\title{
Gribov Copies in the Minimal Landau Gauge: the Influence on Gluon and Ghost Propagators
}

\author{
Attilio Cucchieri* \\ ZiF-Zentrum für Interdisziplinäre Forschung der Universität Bielefeld \\ Wellenberg 1, D-33615 Bielefeld, GERMANY \\ and \\ Fakultät für Physik, Universität Bielefeld \\ Universitätsstrasse, D-33615 Bielefeld, GERMANY
}

August 4, 1997

\begin{abstract}
We study the influence of Gribov copies on gluon and ghost propagators, evaluated numerically in pure $S U(2)$ lattice gauge theory in the minimal Landau gauge. Simulations are done at four different values of $\beta$ (namely $\beta=0,0.8,1.6$ and 2.7 ) and for volumes up to $16^{4}$ (up to $24^{4}$ at $\beta=1.6$ ). For the gluon propagator, Gribov noise seems to be of the order of magnitude of the numerical accuracy, even at very small values of the coupling $\beta$. On the contrary, for the ghost propagator, Gribov noise is clearly observable for the three values of $\beta$ in the strong-coupling regime. In particular, data corresponding to the minimal Landau gauge are always smaller than those obtained in a generic Landau gauge. This result can be qualitatively explained.
\end{abstract}

Gauge theories, being invariant under local gauge transformations, are systems with redundant dynamical variables, which do not represent true dynamical degrees of freedom. The objects of interest are not the gauge fields themselves, but rather the classes (orbits) of gauge-related fields. The elimination of such redundant gauge degrees of freedom is essential for understanding and extracting physical information from these theories. This is usually done by a method called gauge fixing, which is based on the assumption that a gauge-fixing condition can be found which uniquely determines a representative gauge field on each orbit. It was pointed out by Gribov [1] that the standard gauge-fixing conditions used for perturbative calculations do not in fact fix the gauge fields uniquely: for a non-abelian gauge theory, in the Coulomb or in the Landau gauge, there are many gauge equivalent configurations satisfying the Coulomb or Landau transversality condition. The existence of these Gribov copies does not affect the results from perturbation theory, but their elimination could play a crucial role for non-perturbative features of these theories.

One of the celebrated advantages of lattice gauge theories is that the lattice provides a regularization which makes the gauge group compact, so that the Gibbs average of any gauge-invariant quantity is well-defined and therefore gauge fixing is, in principle, not required. However, because

* Present address: Gruppo APE - Dipartimento di Fisica, Università di Roma "Tor Vergata", Via della Ricerca Scientifica 1, I-00133 Roma, ITALY (e-mail: cucchieri@roma2.infn.it). 
of asymptotic freedom, the continuum limit is the weak-coupling limit, and a weak-coupling expansion requires gauge fixing. Moreover, gauge fixing is used in smearing techniques, and is required in order to evaluate quark/gluon matrix elements which can be used to extract non-perturbative results from Monte Carlo simulations. I Thus, one is led to consider gauge-dependent quantities on the lattice as well. Unfortunately gauge fixing on the lattice is afflicted by the same problem of Gribov copies encountered in the continuum case [3, 4, 5, 6]. From a numerical point of view the existence of Gribov copies in the lattice Landau gauge is not surprising. In fact, this gauge condition [0, 8] is imposed by finding a gauge transformation $\{g(x)\}$ which brings the functional

$$
\mathcal{E}_{U}[g]=\frac{1}{8 V} \sum_{\mu, x} \operatorname{Tr}\left[\mathbb{1}-g(x) U_{\mu}(x) g^{\dagger}\left(x+e_{\mu}\right)\right]
$$

to a minimum.2] This functional can be seen as the action of a spin glass model [3], namely a multi-dimensional system characterized by a strong disorder, and therefore it is plausible that one finds a large number of local minima for its energy $\mathcal{E}_{U}$.

Given the appearance of Gribov copies in numerical studies, we need to understand their influence on the evaluation of gauge-dependent quantities. In fact, since usually it is not clear how an algorithm selects among different Gribov copies and we do not know which kind of bias they introduce on the considered quantities (Gribov noise), numerical results using gauge fixing could depend on the gauge-fixing algorithm, making their interpretation conceptually difficult.

Relatively few studies [5, 9, 10, 11, 12, 13, 14] have been done in order to analyze the influence of Gribov copies on lattice quantities. In most cases [10, 11] it was found that Gribov noise is of the same order as the numerical accuracy of the simulations, and that it scales down as a pure statistical error. However, in the so-called maximally abelian gauge, Gribov noise seems to be quite large and to introduce a clear bias on the number of monopoles [9], and on the value of the abelian string tension' 12, 13. Finally, in the case of $U(1)$ lattice gauge theories, it has been shown numerically [5, 14] that the photon propagator is strongly affected by Gribov noise and that only averages taken on absolute minima of the minimizing functional - the $U(1)$-analogue of $\mathcal{E}_{U}[g]$ defined in eq. (四) - reproduce the theoretical predictions. This suggests that the same could happen for the gluon and the ghost propagators.

In this work we present an extensive study of Gribov noise for gluon and ghost propagators at four different values of $\beta$, i.e. $\beta=0,0.8,1.6$ and 2.7. Although these propagators are non-gaugeinvariant quantities, the study of their infra-red behavior provides a powerful tool for increasing our understanding of QCD and, in particular, for gaining insight into the physics of confinement in non-abelian gauge theories. Notice that the first three values of $\beta$ are in the strong-coupling regime, where the number of Gribov copies is higher [6] and Gribov noise, if present, is probably larger and more easily detectable.

Gluon propagators in Landau gauge have been the subject of several numerical studies [8, 16, 17]. However, in these works Gribov copies were not taken into account. In reference [11] Gribov noise has been estimated for the electric and the magnetic screening masses - obtained from the behavior of the gluon propagator in the deconfined phase - and found to be of the order of magnitude of the numerical accuracy. Finally, only one numerical study 18 is available for the ghost propagator.

\footnotetext{
1 For a review see [2].

2 This definition applies to $S U(2)$ lattice gauge theory in 4 dimensions. Here $\left\{U_{\mu}(x)\right\}$ is a given (i.e. fixed) thermalized lattice configuration. See Section 2 for notation and definitions.

3 The string tension, as usually defined, is a gauge-invariant quantity. However, in the maximally abelian gauge, the gauge field is projected onto an abelian lattice gauge field, and the string tension constructed in terms of this abelian field is not gauge-invariant. For details see 13, 15.
} 
Also in this case Gribov noise was not directly analyzed. However, since the fluctuations of the data appear to be very small, the authors concluded that, if present, Gribov noise should be a small effect.

\section{Minimal Landau Gauge}

In order to get rid of the problem of spurious gauge copies, Gribov proposed [1] the use of additional gauge conditions. In particular he restricted the physical configuration space to the region

$$
\Omega \equiv\{A: \partial \cdot A=0, \mathcal{M}[A] \geq 0\}
$$

where $\mathcal{M}[A] \equiv-\nabla \cdot D[A]$ is the Faddeev-Popov operator. This region is clearly included in the hyperplane $\Gamma$ of transverse configurations (i.e. $\partial \cdot A=0)$ and is delimited by the so-called first

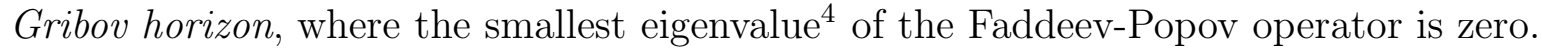

Nowadays we know that the region $\Omega$ is not free of Gribov copies and that conditions stronger than those in (2) should be imposed. In particular the physical configuration space has to be identified with the so-called fundamental modular region $\Lambda$, which is defined (in the continuum) as the set of absolute minima of the functional

$$
E_{A}[g] \equiv\left\|A^{(g)}\right\|^{2} \equiv \frac{1}{2} \sum_{\mu, a} \int d^{4} x\left\{\left[A^{(g)}\right]_{\mu}^{a}(x)\right\}^{2} .
$$

This corresponds to selecting, on a given orbit, the configuration closest to the origin. Of course one has to show that this functional possesses a unique absolute minimum on each orbit. This has been proven in [19]. From these works we also know that $\Omega$ is simply the region of local minima of the functional $E_{A}[g]$, namely it includes $\Lambda$, and is not free of Gribov copies. It has also been shown [20] that in the interior of the fundamental modular region $\Lambda$ the absolute minima are non-degenerate. However, on the boundary of $\Lambda$ there are degenerate absolute minima, and they have to be identified in order to obtain a region truly free of Gribov copies.尸

To eliminate Gribov copies on the lattice we can define a fundamental modular region also in this case. To this end we can look for the absolute minimum of the minimizing functional $\mathcal{E}_{U}[g]$ (minimal Landau gauge), defined in (1), instead of looking for a generic relative minimum. This definition is the lattice analogue of the one used in the continuum [see eq. (3)]. In fact, it is clear that, by choosing the absolute minimum, we select on each gauge orbit the configuration closest to the vacuum $V_{\mu}(x)=\mathbb{1}$. It can also be shown 22, 23] that the set of all minima - local and absolute ones - of the functional $\mathcal{E}_{U}[g]$ is the lattice analogue of the region $\Omega$ introduced by Gribov, namely it is the set of transverse configurations for which the lattice Faddeev-Popov operator $\mathcal{M}[U] \equiv-\nabla \cdot D[U]$ is non-negative. []

Of course, on a finite lattice, the existence of an absolute minimum for $\mathcal{E}_{U}[g]$ is manifest. In fact the gauge orbit is compact and this functional is bounded. However, from a numerical point of view, the search for this absolute minimum is a difficult task. The reason is that eq. (1) represents the energy of a nonlinear sigma-model with variables $g(x)$ taking values on a group [in our case $S U(2)$ ] and, due to this non-linearity, the search for the absolute minimum is highly non-trivial. Moreover,

\footnotetext{
4 The Faddeev-Popov operator has a trivial null eigenvalue, corresponding to a constant eigenvector.

5 This result does not contradict Singer's no-go theorem [21], since the hypothesis of continuity of the gauge-fixing condition is dropped in this approach.

${ }^{6}$ Here $\nabla$ is the lattice four-divergence operator and $D[U]$ is the lattice gauge-covariant derivative.
} 
there are no theoretical predictions that allow us to distinguish a generic relative minimum from the absolute one, except for looking at all minima of the minimizing functional and checking that the (supposed) absolute minimum effectively corresponds to the smallest value of this functional.] The search is therefore a statistical one: enough gauge-fixed gauge copies $C_{j}$ - for each thermalized configuration $C$ - have to be produced so that the probability to get, with a new copy, a value of the functional $\mathcal{E}_{U}[g]$ smaller than all the previous ones is negligible. In particular, we need a statistical criterion in order to decide when to stop this search. Of course, this criterion must balance the necessity of producing enough Gribov copies and the need to keep the computational work limited to a reasonable amount of CPU time. We have decided to proceed in the following way. Given a thermalized configuration $C$, we start by producing five gauge-fixed gauge copies $C_{j}$. The copy characterized by the smallest value for the minimizing functional is chosen as a candidate for the absolute minimum $C_{\min }$. Then we keep doubling the number of gauge-fixed gauge copies $C_{j}$. After each doubling we check if, in one of the new copies, the minimizing functional gets a value smaller than that of the supposed absolute minimum $C_{m i n}$. In the case of a negative result for this check, the search for the absolute minimum is stopped and $C_{\min }$ is used as a chosen candidate for it. In the opposite case, the new gauge-fixed configuration, characterized by the smallest value for the minimizing functional, is chosen as a new candidate for the absolute minimum $C_{\min }$, and we double again the number of Gribov copies. When 320 copies have been produced the search stops anyhow, and the configuration which is the present candidate for $C_{\min }$ is considered as the real absolute minimum. So, for any configuration $C$, a minimum of 10 and a maximum of 320 Gribov copies are produced.

Finally, we consider two different averages: the average considering only the supposed absolute minima (denoted by "am"), which should give us the result in the minimal Landau gauge; and the average considering only the first gauge-fixed gauge copy generated for each configuration (denoted by "fc"). The latter average is the result that we would obtain if Gribov noise were not considered.

\section{Propagators on the Lattice}

We consider a standard Wilson action for $S U(2)$ lattice gauge theory in 4 dimensions with periodic boundary conditions. The symbol $U_{\mu}(x) \in S U(2)$ is used for link variables, $g(x) \in S U(2)$ for site variables, $e_{\mu}$ represents a unit vector in the positive $\mu$ direction, and $V \equiv N^{4}$ is the lattice volume. (Here we always consider lattices with equal sizes in the four directions.) The gauge field is defined as

$$
A_{\mu}(x) \equiv \frac{1}{2}\left[U_{\mu}(x)-U_{\mu}^{\dagger}(x)\right] .
$$

We also define $A_{\mu}^{a}(x) \equiv(1 / 2 i) \operatorname{Tr}\left[A_{\mu}(x) \sigma^{a}\right]$, where $\sigma^{a}$ is a Pauli matrix. Note that $A_{\mu}^{a}(x)$ approaches $(1 / 2)\left[A^{(\text {cont. })}\right]_{\mu}^{a}(x)$ in the continuum limit, where $\left[A^{(\text {cont. })}\right]_{\mu}^{a}(x)$ is the conventional vector potential.

If the configuration $\left\{U_{\mu}(x)\right\}$ is a stationary point of the functional $\mathcal{E}_{U}[g]$, defined in eq. (1), then [7] the lattice divergence of $A_{\mu}^{a}(x)$ is null, namely

$$
(\nabla \cdot A)^{a}(x) \equiv \sum_{\mu} A_{\mu}^{a}(x)-A_{\mu}^{a}\left(x-e_{\mu}\right)=0 \quad \forall \quad x, a .
$$

This is the lattice formulation of the usual Landau gauge-fixing condition in the continuum. By summing equation (5) over the components $x_{\mu}$ of $x$ with $\mu \neq \nu$, for fixed $\nu$, and using the periodicity

\footnotetext{
7 Recently a method to make an extrapolation in the Gribov noise, namely to extrapolate the data in the limit of an infinite number of gauge-fixed gauge copies, has been introduced [13]. An extension of the present work implementing this extrapolation method will be discussed in a future paper.
} 
of the lattice, it is easy to check [8] that if the Landau gauge-fixing condition is satisfied then the quantities

$$
Q_{\nu}\left(x_{\nu}\right) \equiv \sum_{\mu \neq \nu} \sum_{x_{\mu}} A_{\nu}(x) \quad \nu=1, \ldots, 4
$$

are constant, i.e. independent of $x_{\nu}$.

With this notation the lattice space-time gluon propagator is given by

$$
D_{\mu \nu}^{a b}(x-y) \equiv\left\langle A_{\mu}^{a}(x) A_{\nu}^{b}(y)\right\rangle
$$

To go to momentum space we can use formula (3.1a) in reference [22] and obtain

$$
\begin{aligned}
D(0) & \equiv \frac{1}{12 V} \sum_{\mu, a}\left\langle\left[\sum_{x} A_{\mu}^{a}(x)\right]^{2}\right\rangle \\
D(k) & \equiv \frac{1}{9 V} \sum_{\mu, a}\left\langle\left\{\left[\sum_{x} A_{\mu}^{a}(x) \cos (2 \pi k \cdot x)\right]^{2}+\left[\sum_{x} A_{\mu}^{a}(x) \sin (2 \pi k \cdot x)\right]^{2}\right\}\right\rangle
\end{aligned}
$$

(Here $\mu$ goes from 1 to 4 , and $k$ has components $k_{\mu}$ given by $k_{\mu} N \equiv 0,1,2, \ldots, N-1$, where $N$ is the lattice size.) Notice that $D(0)$ is not given by $D(k)$ at $k=0$. The difference is due to the Landau gauge condition - the continuum-like condition as in equation (5) — which in momentum space reads

$$
\sum_{\mu} p\left(k_{\mu}\right) \widetilde{A}_{\mu}^{a}(k) \equiv 2 \sum_{\mu} \sin \left(\pi k_{\mu}\right) \widetilde{A}_{\mu}^{a}(k)=0
$$

If $k \neq 0$ we obtain that only three of the four Lorentz components of $\widetilde{A}^{a}(k)$ - and therefore of $A^{a}(x)$ - are independent: this explains the factor 9 (instead of 12) in equation (9).

The zero three-momentum-space gluon propagator is defined as 8, 16]

$$
D(0, d) \equiv \frac{N}{9 V} \sum_{t, i, a}\left\langle\mathcal{A}_{i}^{a}(t) \mathcal{A}_{i}^{a}(t+d)\right\rangle,
$$

where invariance under space-time translations is used in order to improve statistics. p Here $i$ goes from 1 to 3 (the three directions orthogonal to the time direction, i.e. $i=4$ ) and

$$
\mathcal{A}_{i}^{a}(t) \equiv \sum_{\vec{x}} A_{i}^{a}(\vec{x}, t)
$$

Notice that, if we denote by $\phi_{i}^{a}$ the zero-momentum component of the gluon field $A_{i}^{a}(x)$, namely

$$
\phi_{i}^{a} \equiv \frac{1}{V} \sum_{x} A_{i}^{a}(x)
$$

(where $\mathrm{i}=1,2,3,4$ ), then we can write

$$
\mathcal{A}_{i}^{a}(t) \equiv \frac{V}{N} \phi_{i}^{a}+\left[\mathcal{A}^{(0)}\right]_{i}^{a}(t)
$$

and the relation

$$
\sum_{t}\left[\mathcal{A}^{(0)}\right]_{i}^{a}(t)=0
$$

\footnotetext{
${ }^{8}$ The normalization in (11) has been chosen so that the first term in equations (16) and (17) can be directly related to the zero-momentum gluon propagator in eq. (18).
} 
holds for any $i$ and $a$. Thus we obtain, for the zero three-momentum-space gluon propagator, the expression

$$
\begin{aligned}
D(0, d) & =\frac{V}{9} \sum_{i, a}\left\langle\left(\phi_{i}^{a}\right)^{2}\right\rangle+\frac{N}{9 V} \sum_{t, i, a}\left\langle\left[\mathcal{A}^{(0)}\right]_{i}^{a}(t)\left[\mathcal{A}^{(0)}\right]_{i}^{a}(t+d)\right\rangle \\
& \equiv \Phi^{2}+D^{(0)}(0, d) .
\end{aligned}
$$

Clearly, the contribution of the constants $\phi_{i}^{a}$ to the propagator is considerably enhanced by the volume factor in the first term. The effect of the positive constant $\Phi^{2}$ in eq. (17) on masses obtained in numerical lattice simulations has been recently pointed out in reference [24]. Here we evaluate both the total zero three-momentum gluon propagator, as defined in (11), and the propagator $D^{(0)}(0, d)$ defined in (16) and (17).

Let us observe that a nonzero value for the constants $\phi_{i}^{a}$ is related to the use of periodic boundary conditions and to the finiteness of the volume. In fact, with free boundary conditions, these constants are identically null, even on a finite lattice, after the Landau gauge condition is imposed [25]. Moreover, in the periodic case, it has been proven [22, 26] that these constants must go to zero as the volume increases; more exactly, one obtains $\phi_{i}^{a} \lesssim 1 / N$ as the lattice size $N$ goes to infinity. This bound is a consequence only of the positivity of the Faddeev-Popov matrix in the lattice Landau gauge. Thus, a finite nonzero value of $\phi_{i}^{a}$ is a lattice artifact due to finite volume and translational invariance. Note that this does not imply that $\Phi^{2}$ should be zero in the infinite volume. However, Zwanziger proved [22, 23, 26] that, in the infinite-volume limit, the zero four-momentum gluon propagator $D(0)$, which can be written as

$$
D(0)=\frac{V}{12} \sum_{\mu, a}\left\langle\left(\phi_{\mu}^{a}\right)^{2}\right\rangle,
$$

and is clearly related to $\Phi^{2}$, is less singular at momentum $k=0$ than $k^{-2}$ and that, very likely, it does vanish as rapidly as $k^{2}$. Thus, the infinite-volume limit of $\Phi^{2}$ is related to the infra-red behavior of the gluon propagator, a problem still not settled.

Finally, following [18, 23], we define the space-time ghost propagator as

$$
G(x-y) \delta^{a b} \equiv\left\langle\left(\mathcal{M}^{-1}\right)_{x y}^{a b}[U]\right\rangle
$$

Going to momentum space and taking the trace in color space, we obtain

$$
G(k)=\frac{1}{3 V} \sum_{x, y} e^{-2 \pi i k \cdot(x-y)} \sum_{a}\left\langle\left(\mathcal{M}^{-1}\right)_{x y}^{a a}[U]\right\rangle .
$$

When the lattice Landau gauge is fixed, the Faddeev-Popov matrix is symmetric and semi-positivedefinite [23], and we can write

$$
\mathcal{M}[U]=-(\nabla \cdot D[U])=-(D[U] \cdot \nabla) .
$$

(For an explicit definition of the matrix $\mathcal{M}_{x y}^{a b}[U]$ see eq. (B.18) in reference [23].) In particular, this matrix is positive-definite in the subspace orthogonal to constant vectors. Therefore, it can be inverted by using a standard conjugate-gradient method (CG), provided that we work in this subspace. Moreover, since we use this method in order to evaluate the Faddeev-Popov propagator,

\footnotetext{
${ }^{9}$ Notice that here $\mu$ goes from 1 to 4 , while in eq. (16) the index $i$ goes from 1 to 3 .
} 
we need to impose this restriction in a way that preserves translational invariance for the lattice. This is not a problem if the source $\psi^{a}(x)$ and the initial guess of the solution for the CG-method have zero constant mode [18, 27]. As a source we have used

$$
\psi^{a}(x)=\delta^{a c} e^{-2 \pi i k \cdot x},
$$

where $c$ has a fixed value $(1,2$ or 3$)$ and $k \neq 0$. In this way, the condition $\sum_{x} \psi^{a}(x)=0$ is automatically imposed, and at the same time we save computer time by evaluating "half" of the Fourier transform in eq. (20).

\section{$3 \quad$ Numerical Simulations and Results}

To thermalize the gauge configuration $\left\{U_{\mu}(x)\right\}$ at a fixed value of the coupling $\beta$ we use a hybrid overrelaxed (HOR) algorithm [28]. This algorithm is able to speed up numerical simulations in the case of pure lattice gauge theory: in fact, an overall efficiency gain of up to a factor 10 has been observed [29] [for the $S U(2)$ case] with respect to the heat-bath algorithm, and its dynamic critical exponent $\mathrm{H}^{\mathrm{W}}$ has been found [31] to be equal to 1.0(1) [again in the $S U(2)$ case]. The HOR algorithm is also easy to be implemented: $m$ microcanonical (or energy-conserving) update sweeps are done, followed by one standard local ergodic update (heat-bath sweep) of the lattice. In order to optimize the efficiency of the heat-bath code, we implement two different $S U(2)$ generators (methods 1 and 2 described in Appendix A of [32], with $h_{\text {cutoff }}=2$ ). In our case we did not tune the value of $m$; following [33] we fixed $m=N / 2$. However, for all the pairs $(\beta, N)$, we evaluated the integrated autocorrelation tim ' for the Wilson loops $W(l, l)$ with $l=1,2,4, \ldots, N / 2$, and for the Polyakov loop $P$ (denoted respectively by $\tau_{\text {int }, W_{l}}$ and $\tau_{\text {int,P }}$ ). For all pairs and observables, except for $P$ at $\beta=2.7$, we obtained $\tau_{\text {int }} \lesssim 1$. Note that $\tau_{\text {int }}=0.5$ indicates that two successive configurations generated in the Monte Carlo simulation are independent. The situation is different at $\beta=2.7$, where there is clearly a slow mode associated with the Polyakov loop $P$, a result in agreement with the analysis in reference [34]. At any rate, also in this case we obtained $\tau_{\text {int, } P} \lesssim 20$ for all lattice sizes. Since, for all pairs $(\beta, N)$ and for all quantities, the integrated autocorrelation time is much smaller than the number of sweeps between two consecutive configurations used for evaluating the propagators (see Table 1), we can conclude that these configurations are essentially statistically independent. For the lattice $8^{4}$ at $\beta=2.7$ we also made a comparison between the heat-bath and the HOR algorithm. We obtained $\tau_{\text {int }, P}=225 \pm 68$ in the first case and $\tau_{\text {int, } P}=12.5 \pm 1.2$ in the second one (see [27] for details). Since, in our case, the deterministic update of a link is almost five times faster than the heat-bath one, and "one sweep" of the lattice for the HOR algorithm means $m=4$ microcanonical sweeps followed by one heat-bath sweep, we gain a real (i.e. CPU-time) factor of order 10, in agreement with [29].

For the numerical gauge fixing we use the so-called stochastic overrelaxation algorithm [35], which has dynamic critical exponent $z$ equal to 1 (see [36]). In all our simulations we stop the gauge fixing when the condition

$$
\frac{1}{V} \sum_{x, a}\left[(\nabla \cdot A)^{a}(x)\right]^{2} \leq 10^{-12}
$$

\footnotetext{
10 For a definition of dynamic critical exponent see for example 30.

11 For a definition see [30]. To evaluate the integrated autocorrelation time we use an automatic windowing procedure [30] with two different window factors (6 and 15). We also employ a method [33] based on a comparison between the naive statistical error with a jack-knife binning error [7]. In all cases we checked that these three estimates are in agreement.
} 
is satisfied. This is equivalent [36] to fixing the minimizing functional up to about one part in $10^{12}$, and it is sufficient in order to distinguish two different lattice Gribov copies [3, 4, 5, 6]. In the final gauge-fixed configuration we also evaluate

$$
Q \equiv \frac{1}{12 N} \sum_{\nu} \sum_{x_{\nu}, a}\left[Q_{\nu}^{a}\left(x_{\nu}\right)-\widehat{Q}_{\nu}^{a}\right]^{2}\left[\widehat{Q}_{\nu}^{a}\right]^{-2}
$$

where $\widehat{Q}_{\nu} \equiv(1 / N) \sum_{x_{\nu}} Q_{\nu}\left(x_{\nu}\right)$, and $Q_{\nu}\left(x_{\nu}\right)$ has been defined in eq. (6). This quantity should be zero when the configuration is gauge-fixed, and it is a good estimator of the quality of the gauge fixing. As in reference [36], we found that the stochastic overrelaxation algorithm is very efficient in fighting critical slowing-down [30], and in making the quantities $Q_{\nu}\left(x_{\nu}\right)$ constant.

In Table 1 we report, for each pair $(\beta, N)$, the parameters used for the simulations. Overall, we have considered about 2000 configurations (gauge orbits) and 40,000 Gribov copies. In all our runs we have started from a randomly chosen lattice gauge configuration.

From tests conducted at lattice sizes up to $8^{4}$, we noticed a strong violation of rotational invariance, as is expected for small lattices and non-improved actions. Therefore, we decided to evaluate the four-momentum-space gluon and ghost propagators by considering only values of $k$ with three of the four components equal to zero, namely $k=\left(0,0,0, k_{4}\right)$.

Finally, for the ghost propagator at $\beta=1.6$, data have been collected only up to the volume $16^{4}$, due to limitations on the memory of the available computers.

Computations were performed on several IBM RS-6000/250-340 workstations at New York University.

\subsection{Gluon Propagator}

In Table 2 we show the results (averages "am" and "fc") for the four-momentum-space gluon propagator $D(k)$ as a function of $N k_{4}$. The data corresponding to the minimal Landau gauge (absolute minima) are in complete agreement, within statistical errors, with those obtained in a generic Landau gauge. This happens even at $\beta=0$, where the number of Gribov copies is very large (see Table 11). Clearly for the zero three-momentum-space gluon propagator the result is the same: Gribov noise, if present, seems to be irrelevant compared to the statistical fluctuations. This is the case for both propagators, namely $D(0, d)$ defined in eq. (11) and $D^{(0)}(0, d)$ defined in eq. (16) and (17).

However, a few interesting observations can be made about these data.

First of all, from Table 2 we can see that, in the strong-coupling regime, the infra-red behavior of the gluon propagator is completely different from that at $\beta=2.7$, where it grows very fast as the momentum goes to zero. In particular, at $\beta=0$, this propagator is clearly decreasing as $k_{4}$ goes to zero. At $\beta=0.8$ we see an almost constant propagator. Finally, at $\beta=1.6$ the propagator is increasing as $k_{4}$ goes to zero for the lattice size $N=8$, while it is constant or slightly decreasing with $k_{4}$ for $N=16$ and $N=24$. Also the behavior of zero-momentum gluon propagator $D(0)$ as a function of the lattice size $N$ is very $\beta$-dependent. In fact, at $\beta=0$, it decreases as $N$ increases, and from our data it is not clear if its value would be zero, or a finite nonzero constant, in the limit of $N$ going to infinity. On the contrary, at $\beta=2.7$, this quantity is increasing with the lattice size. Finally, at the two intermediate values of $\beta$ we see a very small volume dependence.

Related to these observations is the fact that, in the strong-coupling regime, the gluon propagator $D(0, d)$ is not always positive (see Figure 1). This is a manifest violation of reflection positivity 22,37 . 
Finally, looking at Figure 2, it is clear that the positiveness of $D(0, d)$ at $\beta=2.7$ is only due to the large value of the positive constant in eq. (17), as predicted in reference [24, Fig. 1].

\subsection{Ghost Propagator}

Results for the ghost propagator are reported in Table 3. In this case it can be noticed that the data obtained in the minimal Landau gauge (average "am") are constantly smaller than or equal to the corresponding "fc"-data. ${ }^{[2}$ Moreover, when the statistics are good, this difference is larger than the numerical uncertainties, especially at small values of $N k_{4}$. The only case in which this does not happen is $\beta=2.7$. However, in this case, even for $N=16$, almost no Gribov copies were produced, and therefore we cannot expect a difference between the two sets of data (see Table 11). At any rate, Gribov noise can clearly be observed for the three strong-coupling values of $\beta$.

This result can be qualitatively explained. In fact, as we said in Section 1, the smallest nontrivial eigenvalue $\lambda_{\min }$ of the Faddeev-Popov operator $\mathcal{M}[U]$ goes to zero as the first Gribov horizon (i.e. the boundary of the region $\Omega$ ) is approached. So, $\lambda_{\min }$ can be interpreted as a sort of distance between the configuration and $\partial \Omega$. Since the fundamental modular region $\Lambda$ is included in $\Omega$, the absolute minimum, which belongs to $\Lambda$, should be "farther" from the the boundary of $\Omega$ than a generic relative minimum. Thus, the absolute minimum configuration should correspond to a value of $\lambda_{\text {min }}$ larger - in average - than the value obtained in a generic relative minimum. [35 This would imply [see eq. (20)] a ghost propagator smaller (in average) at the absolute minimum, as observed in Table 3.

Recently, Zwanziger proposed [23] a modification of the $S U(N)$-Yang-Mills action which effectively constrains the functional integral to the fundamental modular region $\Lambda$ in minimal Landau gauge. The lattice partition function of this model can be directly evaluated, by the saddle-point method, in the thermodynamic limit. From this theory, it is possible to conclude [23] that, in four Euclidean dimensions, the lattice ghost propagator has a dipole singularity at zero momentum. In order to check the infra-red behavior of the ghost propagator we divided $G(k)$ (data in Table 3 , average "am") by the corresponding free propagator

$$
G^{\text {free }}(k) \equiv\left[4 \sum_{\mu} \sin ^{2}\left(\pi k_{\mu}\right)\right]^{-1} \equiv 1 / p^{2}(k)
$$

In Figure 3 we plot the results for the four different values of $\beta$ and lattice size $N=16$. (Similar results can be obtained for different lattice sizes). From these data it is clear that $G(k)$ diverges faster than $1 / p^{2}(k)$ as $k$ goes to zero. In order to analyze this singularity we have tried a fit of the data in Table 3 (average "am") using the Ansatz

$$
G(k)=a p^{-2}(k)+b p^{-4}(k)+\ldots,
$$

as in reference [18]. In all cases we have found that the fit with $b$ set to zero is very poor. When the dipole singularity is included, the fit improves and we have $a \gtrsim b$. However, for large lattice sizes and small momenta, the fit always overestimates the numerical values of the data. This is in agreement with results in reference [18], and suggests a singularity at zero momentum smaller than $p^{-4}(k)$. As a second attempt we tried a fit四 to the Ansatz

$$
G(k)=a\left[p^{-2}(k)\right]^{s} .
$$

\footnotetext{
12 This is not the case only for $\beta=1.6, N=16$ and $N k_{4}=1$.

13 A numerical study of this eigenvalue and its dependence on Gribov copies has been done in reference [27] and will be reported in a future paper [38]. Results seem to confirm the scenario described here.

${ }^{14}$ In both cases the fit has been done using a mathematica program implementing the singular-value-decomposition method (see for example [39]).
} 
In this case the fit is in general quite good. Finally, in order to probe the small-momentum limit, we evaluate the exponent $s$ in eq. (27) using only data corresponding to the two smallest momenta available for each lattice size. From the results, reported in Table 4 , it can be noticed that $s$ decreases as the lattice size increases, while it increases as $\beta$ increases. The last result is also clear in Figure 3 .

\section{Conclusions}

Our data show absence of Gribov noise for the gluon propagator and a nonzero Gribov noise for the ghost propagator. In the latter case, the effect is small but clearly detectable for the values of $\beta$ in the strong-coupling region. The fact that this noise is not observable at $\beta=2.7$ seems to us to be related only to the small volumes considered here. Of course this hypothesis should be checked numerically. This is, at the moment, beyond the limits of our computational resources. We stress that this is the first case of evidence of Gribov noise in a "truly" non-abelian simulation. To date, in fact, Gribov noise was observed only in simulations for the $U(1)$ case [5, 14], or using maximally abelian gauge [9, 12, 13].

As for the infra-red behavior of these two propagators, the data for the ghost propagator show a pole "between" the zeroth-order perturbative behavior $p^{-2}(k)$ — valid at large momenta — and the $p^{-4}(k)$ singularity predicted in [23]. However, in this case, the volumes used do not really allow an analysis of the small-momentum limit, and this analysis is complicated by the fact that the ghost propagator cannot be evaluated at zero momentum. For the gluon propagator, at least in the strongcoupling regime, the data are quite interesting, showing a propagator decreasing as the momentum

goes to zero. This anomalous behavior, predicted in [1, 22, 23], is still observable at $\beta=1.6$, if large volumes are considered. Related to this behavior is the violation of reflection positivity observable in Figure 1. Finally, our data show that the behavior of the zero three-momentum-space gluon propagator is strongly affected by the zero-momentum modes of the gluon field [24]. We think that these results deserve a more accurate numerical analysis which will be presented in a separate paper 40.

\section{Acknowledgements}

I am indebted to D.Zwanziger for suggesting this work to me. I also would like to thank him, G.Dell'Antonio, T.Mendes, V.K.Mitryushkin, S.Petrarca, M.Schaden and B.Taglienti for valuable discussions and suggestions, and Ph. de Forcrand for e-mail correspondence.

Part of this work has been done at the University of Rome "Tor Vergata"; I thank the Physics Department, and in particular R.Petronzio and the APE group, for the hospitality.

I also thank for the hospitality the Physics Department of the University of Bielefeld and the Center for Interdisciplinary Research $(\mathrm{ZiF})$ at Bielefeld, where this work has been finished.

\section{References}

[1] V.N.Gribov, Nucl.Phys. B139 (1978) 1.

[2] G.C.Rossi, Nucl.Phys. B (Proc. Suppl.) 53 (1997) 3.

[3] E.Marinari, C.Parrinello and R.Ricci, Nucl.Phys. B362 (1991) 487. 
[4] Ph. de Forcrand et al., Nucl.Phys. B (Proc. Suppl.) 20 (1991) 194; J.E.Mandula and M.C.Ogilvie, Phys.Rev. D41 (1990) 2586; J.E.Hetrick et al., Nucl.Phys. B (Proc. Suppl.) 26 (1992) 432; A.Nakamura and M.Mizutani, Vistas in Astronomy 37 (1993) 305.

[5] Ph. de Forcrand and J.E.Hetrick, Nucl.Phys. B (Proc. Suppl.) 42 (1995) 861.

[6] P.Marenzoni and P.Rossi, Phys.Lett. B311 (1993) 219.

[7] K.G.Wilson, Recent Developments in Gauge Theories Proc. NATO Advanced Study Institute (Cargése, 1979), eds. G. 't Hooft et al. (Plenum Press, New York-London, 1980).

[8] J.E.Mandula and M.Ogilvie, Phys.Lett. B185 (1987) 127.

[9] S.Hioki et al., Phys.Lett. B271 (1991) 201.

[10] M.L.Paciello et al., Phys.Lett. B289 (1992) 405; M.L.Paciello et al., Phys.Lett. B341 (1994) 341; L.Conti et al., Phys.Lett. B373 (1996) 164.

[11] U.M.Heller, F.Karsch and J.Rank, Phys.Lett. B355 (1995) 511.

[12] G.S.Bali et al., Nucl.Phys. B (Proc. Suppl.) 42 (1995) 852.

[13] G.S.Bali et al., Phys.Rev. D54 (1996) 2863.

[14] A.Nakamura and M.Plewnia, Phys.Lett. B255 (1991) 274; V.G.Bornyakov et al., Nucl.Phys. B (Proc. Suppl.) 34 (1994) 802.

[15] L. Del Debbio et al., Nucl.Phys. B (Proc. Suppl.) 53 (1997) 141.

[16] R.Gupta et al., Phys.Rev. D36 (1987) 2813; P.Marenzoni et al., Phys.Lett. B318 (1993) 511; C.Bernard, C.Parrinello and A.Soni, Phys.Rev. D49 (1994) 1585; P.Marenzoni, G.Martinelli and N.Stella, Nucl.Phys. B455 (1995) 339.

[17] A.Nakamura et al., Gluon Propagators and Confinement, hep-lat/9506024, published in RCNP Confinement 1995; H.Aiso et al., Nucl.Phys. B (Proc. Suppl.) 53 (1997) 570; F.Gutbrod, A Study of the Gluon Propagator in SU(2) Lattice Gauge Theory, DESY-96-252 preprint.

[18] H.Suman and K.Schilling, Phys.Lett. B373 (1996) 314.

[19] M.A.Semenov-Tyan-Shanskii and V.A.Franke, Zapiski Nauchnykh Seminarov Leningradskogo Otdeleniya Matematicheskogo Instituta im. V.A.Steklova AN SSSR 120 (1982) 159 [English translation: Journ.Sov.Math. 34 (1986) 1999]; G.Dell'Antonio and D.Zwanziger, Commun.Math.Phys. 138 (1991) 291.

[20] P. van Baal, Nucl.Phys. B369 (1992) 259; P. van Baal, Global Issues in Gauge Fixing, hepth/9511119, published in the Proceedings of the QCD Workshop, Trento (Italy) 1995.

[21] I.M.Singer, Commun.Math.Phys. 60 (1978) 7; T.P.Killingback, Phys.Lett. B138 (1984) 87.

[22] D.Zwanziger, Nucl.Phys. B364 (1991) 127.

[23] D.Zwanziger, Nucl.Phys. B412 (1994) 657. 
[24] V.K.Mitryushkin, Phys.Lett. B390 (1997) 293.

[25] M.Schaden and D.Zwanziger, Horizon Condition Holds Pointwise on Finite Lattice with Free Boundary Conditions, hep-th/9410019, published in the Proceedings of the Workshop on Quantum Infrared Physics, Paris (France) 6-10 Jun 1994.

[26] D.Zwanziger, Phys.Lett. B257 (1991) 168.

[27] A.Cucchieri, Numerical Results in Minimal Lattice Coulomb and Landau Gauges: ColorCoulomb Potential and Gluon and Ghost Propagators, PhD thesis, New York University (May 1996).

[28] F.R.Brown and T.J.Woch, Phys.Rev.Lett. 58 (1987) 2394; S.L.Adler, Nucl.Phys. B (Proc. Suppl.) 9 (1989) 437; U.Wolff, Nucl.Phys. B (Proc. Suppl.) 17 (1990) 93; U.Wolff, Phys.Lett. B288 (1992) 166; U.Wolff, Int.J.Mod.Phys. C4 (1993) 451.

[29] K.M.Decker and Ph. de Forcrand, Nucl.Phys. B (Proc. Suppl.) 17 (1990) 567.

[30] A.D.Sokal, Monte Carlo Methods in Statistical Mechanics: Foundations and New Algorithms, Cours de Troisième Cycle de la Physique en Suisse Romande (Lausanne, June 1989).

[31] M.Lüscher et al., Nucl.Phys. B389 (1993) 247.

[32] R.G.Edwards et al., Nucl.Phys. B380 (1992) 621.

[33] G. de Divitiis et al., Nucl.Phys. B437 (1995) 447.

[34] M.Grabenstein and K.Pinn, Phys.Rev. D50 (1994) 6998.

[35] Ph. de Forcrand and R.Gupta, Nucl.Phys. B (Proc. Suppl.) 9 (1989) 516.

[36] A.Cucchieri and T.Mendes, Nucl.Phys. B471 (1996) 263; A.Cucchieri and T.Mendes, Nucl.Phys. B (Proc. Suppl.) 53 (1997) 811; A.Cucchieri and T.Mendes, Critical Slowing-Down in SU(2) Landau Gauge-Fixing Algorithms (II): the Four-Dimensional Case, to be submitted to Nucl.Phys. B.

[37] L. von Smekal, A.Hauck and R.Alkofer, A Solution to Coupled Dyson-Schwinger Equations for Gluons and Ghosts in Landau Gauge, hep-ph/9707327.

[38] A.Cucchieri, Numerical Study of the Fundamental Modular Region in the Minimal Landau Gauge, in preparation.

[39] W.H.Press et al., Numerical Recipes (Cambridge University Press, Cambridge, 1992, second edition).

[40] A.Cucchieri, Infra-Red Behavior of the Gluon Propagator in Lattice Landau Gauge, submitted to Phys.Lett. B. 


\begin{tabular}{||c|c|c|c|c|c|c|c||}
\hline \hline$\beta$ & $N$ & config. & th. sweeps & sweeps & $p$ & copies & fc $\neq$ am \\
\hline 0.0 & 4 & $400(304)$ & 10 & 10 & 0.84 & $4220(1127)$ & 159 \\
\hline 0.0 & 6 & $200(200)$ & 20 & 10 & 0.875 & $4060(3121)$ & 167 \\
\hline 0.0 & 8 & $200(200)$ & 10 & 10 & 0.915 & $6120(6071)$ & 179 \\
\hline 0.0 & 10 & $100(100)$ & 20 & 10 & 0.945 & $3850(3840)$ & 89 \\
\hline 0.0 & 12 & $80(80)$ & 20 & 10 & 0.945 & $1940(1936)$ & 71 \\
\hline 0.0 & 14 & $20(20)$ & 20 & 10 & 0.95 & $590(590)$ & 19 \\
\hline 0.0 & 16 & $10(10)$ & 20 & 10 & 0.96 & $510(506)$ & 9 \\
\hline \hline 0.8 & 8 & $200(200)$ & 1000 & 100 & 0.875 & $6950(6627)$ & 177 \\
\hline 0.8 & 12 & $22(22)$ & 1375 & 125 & 0.9 & $1240(1239)$ & 20 \\
\hline 0.8 & 16 & $9(9)$ & 1100 & 100 & 0.94 & $140(140)$ & 8 \\
\hline \hline 1.6 & 8 & $100(100)$ & 1100 & 100 & 0.865 & $1910(1545)$ & 85 \\
\hline 1.6 & 16 & $20(20)$ & 1650 & 150 & 0.92 & $910(905)$ & 19 \\
\hline 1.6 & 24 & $7(7)$ & 1650 & 150 & 0.91 & $290(290)$ & 6 \\
\hline \hline 2.7 & 8 & $400(47)$ & 2200 & 200 & 0.8 & $4020(494)$ & 17 \\
\hline 2.7 & 12 & $158(25)$ & 2200 & 200 & 0.85 & $1600(208)$ & 11 \\
\hline 2.7 & 16 & $100(26)$ & 2200 & 200 & 0.875 & $1000(157)$ & 13 \\
\hline \hline
\end{tabular}

Table 1: The pairs $(\beta, N)$ used for the simulations, the number of configurations (in brackets, the number of configurations for which Gribov copies have been found), the number of sweeps used for thermalization, the number of sweeps between two consecutive configurations used for collecting our data, the parameter $p$ used by the stochastic overrelaxation algorithm, the total number of Gribov copies produced (in brackets, the total number of different Gribov copies), and the number of times the first copy produced was not the absolute minimum copy. 


\begin{tabular}{|c|c|c|c|c|c|c|c|c|c|c|c|}
\hline $\bar{\beta}$ & $\bar{N}$ & stat. & 0 & 1 & 2 & $\overline{3}$ & $\overline{4}$ & $\overline{5}$ & $\overline{6}$ & $\overline{7}$ & $\overline{8}$ \\
\hline$\overline{0.0}$ & $\overline{4}$ & $\overline{\mathrm{am}}$ & $\overline{0.208(0.004)}$ & $0.218(0.003)$ & $0.226(0.006)$ & & & & & & \\
\hline 0.0 & 4 & $\mathrm{fc}$ & $0.207(0.004)$ & $0.221(0.003)$ & $0.229(0.005)$ & & & & & & \\
\hline 0.0 & 6 & $\mathrm{am}$ & $0.185(0.006)$ & $0.189(0.004)$ & $0.214(0.005)$ & $0.219(0.007)$ & & & & & \\
\hline 0.0 & 6 & fc & $0.183(0.006)$ & $0.198(0.004)$ & $0.215(0.005)$ & $0.222(0.007)$ & & & & & \\
\hline 0.0 & 8 & $\mathrm{am}$ & $0.162(0.005)$ & $0.174(0.004)$ & $0.196(0.004)$ & $0.209(0.005)$ & $0.209(0.006)$ & & & & \\
\hline 0.0 & 8 & fc & $0.160(0.005)$ & $0.177(0.004)$ & $0.186(0.004)$ & $0.209(0.005)$ & $0.211(0.006)$ & & & & \\
\hline 0.0 & 10 & $\mathrm{am}$ & $0.154(0.007)$ & $0.168(0.005)$ & $0.195(0.006)$ & $0.211(0.007)$ & $0.202(0.007)$ & $0.22(0.01)$ & & & \\
\hline 0.0 & 10 & fc & $0.157(0.007)$ & $0.172(0.006)$ & $\begin{array}{l}0.196(0.006) \\
\end{array}$ & $0.203(0.007)$ & $0.214(0.007)$ & $0.22(0.01)$ & & & \\
\hline 0.0 & 12 & $\mathrm{am}$ & $0.160(0.007)$ & $0.147(0.005)$ & $0.186(0.007)$ & $0.194(0.007)$ & $0.203(0.008)$ & $0.205(0.007)$ & $0.21(0.01)$ & & \\
\hline 0.0 & 12 & $\mathrm{fc}$ & $0.148(0.007)$ & $0.169(0.006)$ & $0.181(0.007)$ & $0.192(0.006)$ & $0.214(0.008)$ & $0.210(0.007)$ & $0.22(0.01)$ & & \\
\hline 0.0 & 14 & $\mathrm{am}$ & $0.15(0.01)$ & $0.17(0.01)$ & $0.18(0.01)$ & $0.19(0.01)$ & $0.23(0.02)$ & $0.23(0.02)$ & $0.23(0.02)$ & $0.24(0.03)$ & \\
\hline 0.0 & 14 & fc & $0.15(0.01)$ & $0.14(0.01)$ & $0.18(0.02)$ & $0.19(0.01)$ & $0.18(0.01)$ & $0.24(0.02)$ & $0.25(0.02)$ & $0.19(0.02)$ & \\
\hline 0.0 & 16 & $\mathrm{am}$ & $0.12(0.01)$ & $0.15(0.02)$ & $0.17(0.02)$ & $0.16(0.01)$ & $0.19(0.02)$ & $0.21(0.02)$ & $0.20(0.02)$ & $0.21(0.02)$ & $0.20(0.03)$ \\
\hline 0.0 & 16 & fc & $0.12(0.01)$ & $0.16(0.02)$ & $0.15(0.02)$ & $0.22(0.02)$ & $0.20(0.02)$ & $0.22(0.02)$ & $0.20(0.02)$ & $0.23(0.03)$ & $0.21(0.04)$ \\
\hline$\overline{0.8}$ & $\overline{88}$ & $\overline{\mathrm{am}}$ & $0.268(0.008)$ & $0.284(0.007)$ & $0.281(0.007)$ & $0.273(0.006)$ & $0.251(0.009)$ & & & & \\
\hline 0.8 & 8 & fc & $0.281(0.008)$ & $0.293(0.007)$ & $0.281(0.007)$ & $0.268(0.006)$ & $0.26(0.01)$ & & & & \\
\hline 0.8 & 12 & $\mathrm{am}$ & $0.26(0.02)$ & $0.23(0.02)$ & $0.25(0.02)$ & $0.30(0.02)$ & $0.28(0.02)$ & $0.24(0.01)$ & $0.28(0.03)$ & & \\
\hline 0.8 & 12 & fc & $0.25(0.02)$ & $0.27(0.02)$ & $0.26(0.02)$ & $0.30(0.02)$ & $0.26(0.02)$ & $0.25(0.02)$ & $0.28(0.02)$ & & \\
\hline 0.8 & 16 & $\mathrm{am}$ & $0.26(0.04)$ & $0.25(0.02)$ & $0.27(0.02)$ & $0.39(0.05)$ & $0.33(0.04)$ & $0.25(0.02)$ & $0.23(0.03)$ & $0.26(0.03)$ & $0.25(0.04)$ \\
\hline 0.8 & 16 & fc & $0.23(0.02)$ & $0.31(0.04)$ & $0.30(0.04)$ & $0.28(0.04)$ & $0.26(0.03)$ & $0.27(0.04)$ & $0.25(0.01)$ & $0.25(0.03)$ & $0.28(0.04)$ \\
\hline 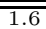 & $\overline{88}$ & $\overline{\mathrm{am}}$ & $\overline{0.64(0.03)}$ & $\overline{0.61(0.02)}$ & $\overline{0.47(0.02)}$ & $\overline{0.31(0.01)}$ & $0.27(0.01)$ & & & & \\
\hline 1.6 & 8 & fc & $0.67(0.03)$ & $0.61(0.02)$ & $0.47(0.01)$ & $0.32(0.01)$ & $0.28(0.01)$ & & & & \\
\hline 1.6 & 16 & $\mathrm{am}$ & $0.50(0.04)$ & $0.55(0.03)$ & $0.68(0.04)$ & $0.49(0.03)$ & $0.47(0.03)$ & $0.33(0.02)$ & $0.32(0.02)$ & $0.29(0.01)$ & $0.31(0.03)$ \\
\hline 1.6 & 16 & $\mathrm{fc}$ & $0.59(0.05)$ & $0.58(0.03)$ & $0.57(0.04)$ & $0.52(0.05)$ & $0.45(0.03)$ & $0.39(0.03)$ & $0.31(0.02)$ & $0.29(0.02)$ & $0.29(0.04)$ \\
\hline 1.6 & 24 & $\mathrm{am}$ & $0.57(0.04)$ & $0.7(0.1)$ & $0.60(0.05)$ & $0.63(0.04)$ & $0.50(0.06)$ & $0.49(0.05)$ & $0.48(0.05)$ & $0.40(0.09)$ & $0.35(0.05)$ \\
\hline 1.6 & 24 & fc & $0.55(0.04)$ & $0.60(0.06)$ & $0.58(0.09)$ & $0.58(0.08)$ & $0.5(0.1)$ & $0.42(0.05)$ & $0.50(0.09)$ & $0.53(0.07)$ & $0.45(0.08)$ \\
\hline$\overline{2.7}$ & $\overline{88}$ & $\overline{\mathrm{am}}$ & $30.3(0.5)$ & 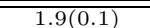 & $0.274(0.005)$ & $0.141(0.002)$ & $0.114(0.003)$ & & & & \\
\hline 2.7 & 8 & $\mathrm{fc}$ & $30.2(0.5)$ & $2.0(0.1)$ & $0.276(0.005)$ & $0.142(0.002)$ & $0.114(0.003)$ & & & & \\
\hline 2.7 & 12 & $\mathrm{am}$ & $59(2)$ & $4.2(0.3)$ & $0.64(0.02)$ & $0.275(0.007)$ & $0.169(0.004)$ & $0.132(0.003)$ & $0.113(0.004)$ & & \\
\hline 2.7 & 12 & fc & $58(1)$ & $4.4(0.3)$ & $0.64(0.02)$ & $0.273(0.007)$ & $0.171(0.004)$ & $0.131(0.003)$ & $0.114(0.004)$ & & \\
\hline 2.7 & 16 & $\mathrm{am}$ & $94(3)$ & $8.2(0.8)$ & $1.19(0.05)$ & $0.47(0.01)$ & $0.253(0.009)$ & $0.183(0.006)$ & $0.144(0.005)$ & $0.123(0.004)$ & $0.116(0.005)$ \\
\hline 2.7 & 16 & fc & $93(3)$ & $8.2(0.7)$ & $1.17(0.04)$ & $0.47(0.01)$ & $0.259(0.009)$ & $0.183(0.006)$ & $0.144(0.005)$ & $0.123(0.004)$ & $0.114(0.005)$ \\
\hline
\end{tabular}

Table 2: The four-momentum-space gluon propagator $D(k)$ [see eq. (8) and (9)] as a function of $N k_{4}$. In all our runs we set $k=\left(0,0,0, k_{4}\right)$. Clearly the same value of $N k_{4}$ for different lattice sizes $N$ does not correspond to the same lattice momentum $p_{4}$ [see eq. (10)]. Since we use periodic boundary conditions, only data for $N k_{4} \leq N / 2$ are reported here. Two different types of statistics are considered: "am" and "fc". (For a definition see the last paragraph in Section 11.) Error bars (in brackets) are one standard deviation. To make the table more readable, data for $N=24, \beta=1.6$ and $k_{4}>8$ are not reported. 


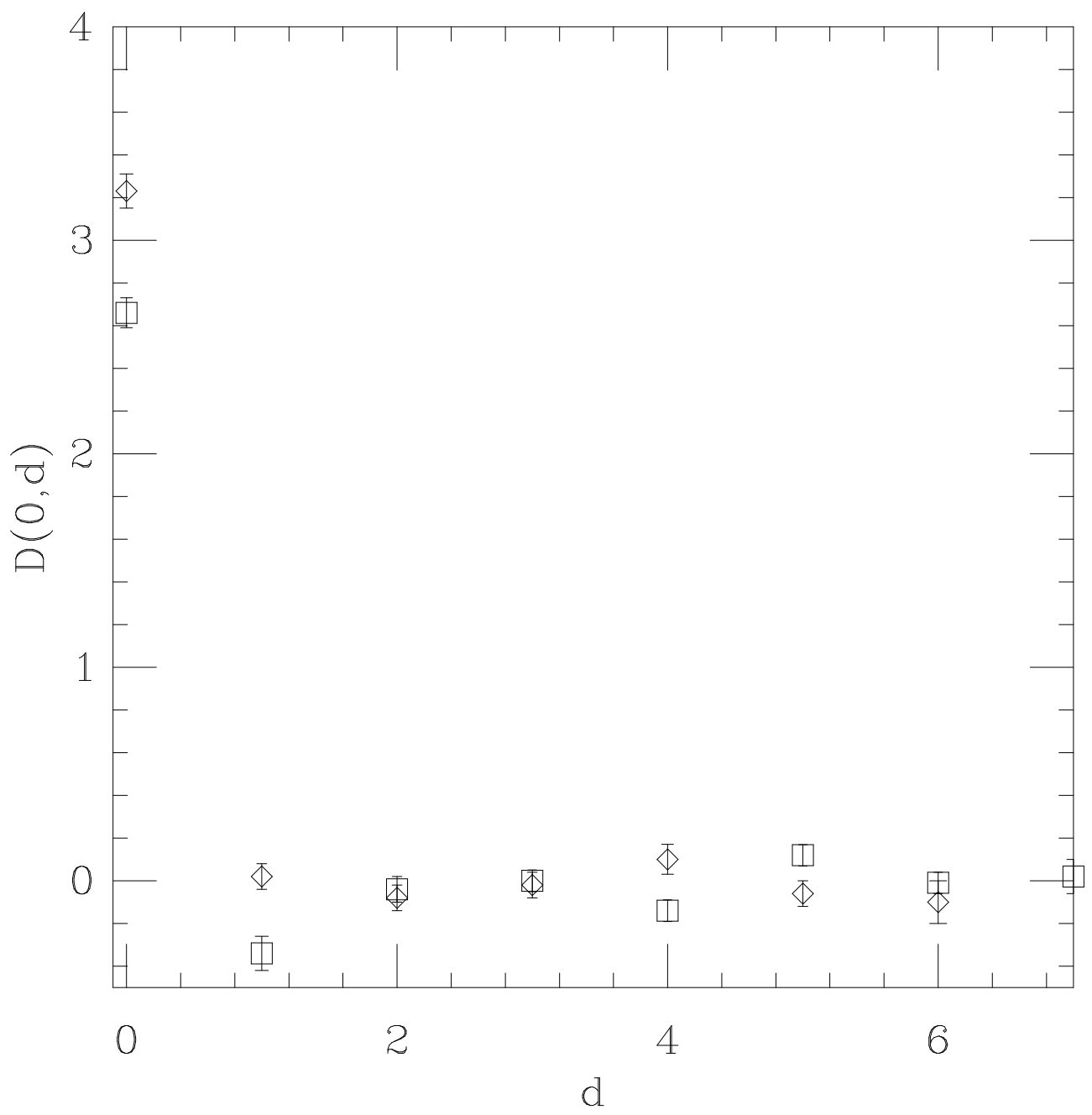

Figure 1: The zero three-momentum-space gluon propagator $D(0, d)$ [see eq. (11)] as a function of the time separation $d$. Since we use periodic boundary conditions, only data for $d \leq N / 2$ are reported here. Data correspond to $V=14^{4}$ at $\beta=0(\square)$, and $V=12^{4}$ at $\beta=0.8(\diamond)$. Here we consider the "fc" statistics (for a definition see the last paragraph in Section 1.) Error bars are one standard deviation. 


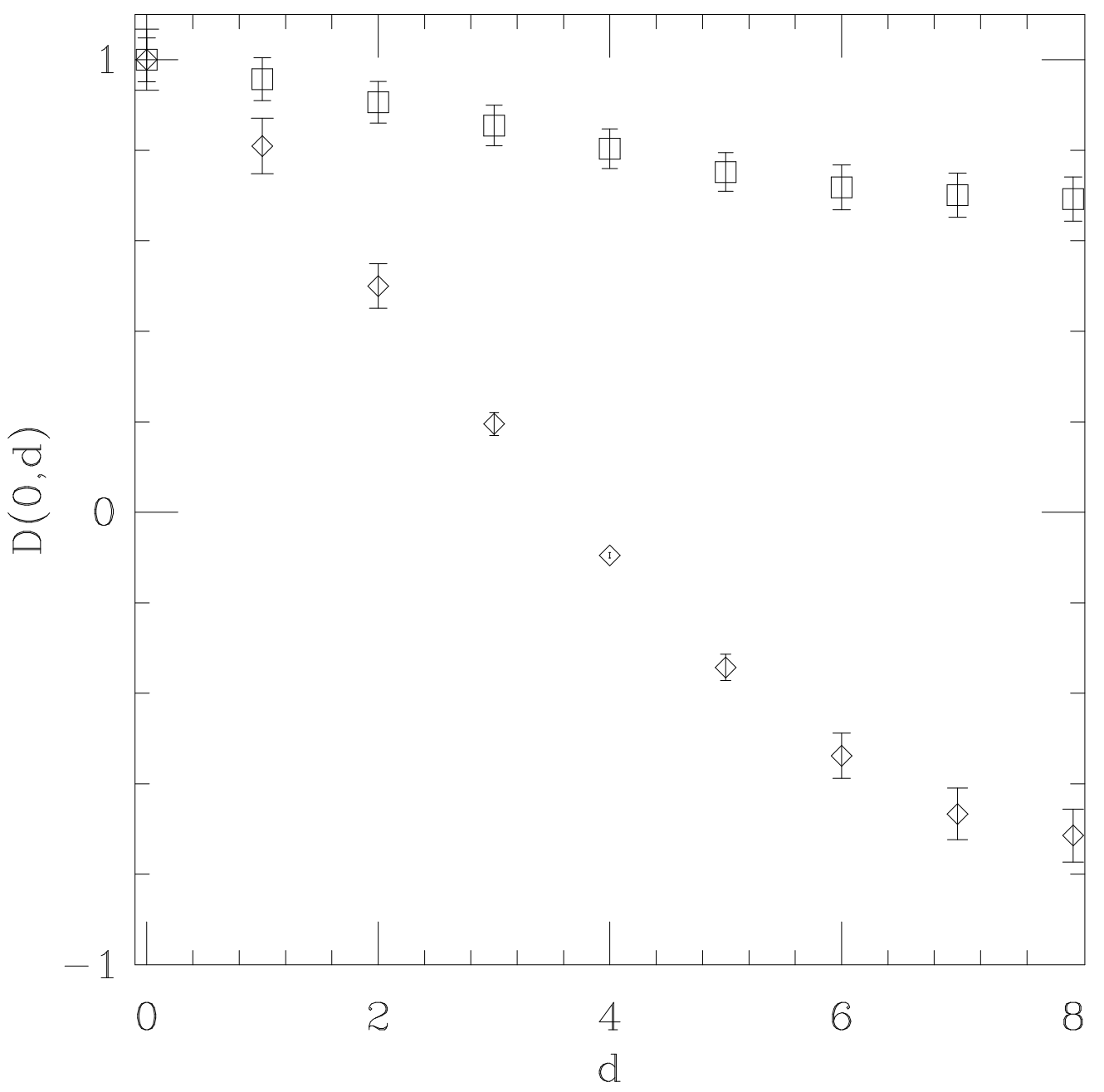

Figure 2: Comparison between the zero three-momentum-space gluon propagators $D(0, d)(\square)$ and $D^{(0)}(0, d)(\diamond)$ [see eqs. (11) and (16)] as a function of the time separation $d$. Since we use periodic boundary conditions, only data for $d \leq N / 2$ are reported here. The data, normalized to one at the zero time-separation, correspond to $V=16^{4}$ and $\beta=2.7$. Here we consider the "fc" statistics (for a definition see the last paragraph in Section ‥ Error bars are one standard deviation. 


\begin{tabular}{|c|c|c|c|c|c|c|c|c|c|c|}
\hline$\beta$ & $\bar{N}$ & stat. & $\overline{1}$ & $\overline{2}$ & $\overline{3}$ & $\overline{4}$ & $\overline{5}$ & $\overline{6}$ & $\overline{7}$ & $\overline{8}$ \\
\hline$\overline{0.0}$ & $\overline{4}$ & $\overline{a m}$ & $2.29(0.03)$ & $\overline{0.894(0.007)}$ & & & & & & \\
\hline 0.0 & 4 & fc & $2.35(0.03)$ & $0.916(0.008)$ & & & & & & \\
\hline 0.0 & 6 & $\mathrm{am}$ & $5.21(0.05)$ & $1.287(0.009)$ & $0.876(0.005)$ & & & & & \\
\hline 0.0 & 6 & $\mathrm{fc}_{\mathrm{c}}$ & $5.54(0.06)$ & $1.34(0.01)$ & $0.906(0.007)$ & & & & & \\
\hline 0.0 & 8 & $\mathrm{am}$ & $9.85(0.08)$ & $2.19(0.02)$ & $1.088(0.005)$ & $0.880(0.005)$ & & & & \\
\hline 0.0 & 8 & $\mathrm{fc}_{\mathrm{C}}$ & $10.19(0.08)$ & $2.23(0.01)$ & $1.102(0.004)$ & $0.889(0.004)$ & & & & \\
\hline 0.0 & 10 & $\mathrm{am}$ & $16.0(0.1)$ & $3.48(0.02)$ & $1.534(0.005)$ & $1.004(0.003)$ & $0.877(0.003)$ & & & \\
\hline 0.0 & 10 & fc & $16.9(0.2)$ & $3.58(0.02)$ & $1.564(0.007)$ & $1.015(0.004)$ & $0.888(0.003)$ & & & \\
\hline 0.0 & 12 & $\mathrm{am}$ & $24.1(0.2)$ & $5.19(0.02)$ & $2.177(0.008)$ & $1.285(0.004)$ & $0.960(0.002)$ & $0.876(0.002)$ & & \\
\hline 0.0 & 12 & fc & $24.6(0.2)$ & $5.28(0.03)$ & $2.204(0.009)$ & $1.296(0.004)$ & $0.968(0.003)$ & $0.883(0.003)$ & & \\
\hline 0.0 & 14 & $\mathrm{am}$ & $33.1(0.3)$ & $7.28(0.04)$ & $2.99(0.01)$ & $1.681(0.007)$ & $1.157(0.004)$ & $0.938(0.003)$ & $0.876(0.003)$ & \\
\hline 0.0 & 14 & fc & $34.8(0.3)$ & $7.54(0.04)$ & $3.07(0.01)$ & $1.706(0.006)$ & $1.170(0.004)$ & $0.948(0.003)$ & $0.888(0.004)$ & \\
\hline 0.0 & 16 & $\mathrm{am}$ & $44.2(0.7)$ & $9.9(0.1)$ & $4.04(0.04)$ & $2.20(0.02)$ & $1.434(0.006)$ & $1.085(0.004)$ & $0.926(0.004)$ & $0.879(0.004)$ \\
\hline 0.0 & 16 & $\mathrm{am}$ & $45.5(0.7)$ & $9.9(0.1)$ & $4.02(0.04)$ & $2.18(0.02)$ & $1.432(0.009)$ & $1.085(0.006)$ & $0.922(0.005)$ & $0.874(0.005)$ \\
\hline 0.8 & 8 & $\mathrm{am}$ & $8.41(0.07)$ & $1.778(0.008)$ & $0.861(0.003)$ & $\overline{0.689(0.002)}$ & & & & \\
\hline 0.8 & 8 & fc & $8.94(0.08)$ & $1.843(0.009)$ & $0.880(0.003)$ & $0.701(0.003)$ & & & & \\
\hline 0.8 & 12 & $\mathrm{am}$ & $20.8(0.1)$ & $4.42(0.03)$ & $1.793(0.009)$ & $1.031(0.004)$ & $0.761(0.003)$ & $0.690(0.002)$ & & \\
\hline 0.8 & 12 & fc & $22.1(0.6)$ & $4.53(0.05)$ & $1.84(0.01)$ & $1.053(0.005)$ & $0.772(0.003)$ & $0.697(0.003)$ & & \\
\hline 0.8 & 16 & $\mathrm{am}$ & $39.3(0.6)$ & $8.47(0.08)$ & $3.37(0.02)$ & $1.800(0.009)$ & $1.160(0.006)$ & $0.866(0.004)$ & $0.732(0.003)$ & $0.693(0.003)$ \\
\hline 0.8 & 16 & fc & $40.6(0.7)$ & $8.7(0.1)$ & $3.43(0.03)$ & $1.82(0.02)$ & $1.18(0.01)$ & $0.875(0.007)$ & $0.738(0.005)$ & $0.698(0.004)$ \\
\hline 1.6 & 8 & $\mathrm{am}$ & $6.61(0.06)$ & $1.335(0.006)$ & $0.632(0.002)$ & $0.503(0.001)$ & & & & \\
\hline 1.6 & 8 & fc & $7.2(0.1)$ & $1.38(0.01)$ & $0.645(0.004)$ & $0.512(0.003)$ & & & & \\
\hline 1.6 & 16 & $\mathrm{am}$ & $32.2(0.5)$ & $6.66(0.07)$ & $2.56(0.02)$ & $1.329(0.005)$ & $0.845(0.003)$ & $0.627(0.002)$ & $0.528(0.001)$ & $0.500(0.001)$ \\
\hline 1.6 & 16 & fc & $32.1(0.3)$ & $6.66(0.04)$ & $2.56(0.01)$ & $1.332(0.006)$ & $0.847(0.003)$ & $0.628(0.002)$ & $0.530(0.001)$ & $0.5004(0.0009)$ \\
\hline 2.7 & 8 & $\mathrm{am}$ & $3.4(0.1)$ & $0.635(0.002)$ & $0.3494(0.0004)$ & $0.2937(0.0003)$ & & & & \\
\hline 2.7 & 8 & fc & $3.4(0.1)$ & $0.636(0.002)$ & $0.3495(0.0005)$ & $0.2938(0.0003)$ & & & & \\
\hline 2.7 & 12 & $\mathrm{am}$ & $7.0(0.2)$ & $1.308(0.005)$ & $0.610(0.001)$ & $0.3939(0.0004)$ & $0.3116(0.0002)$ & $0.2893(0.0002)$ & & \\
\hline 2.7 & 12 & fc & $7.1(0.3)$ & $1.309(0.005)$ & $0.610(0.001)$ & $0.3939(0.0004)$ & $0.3117(0.0002)$ & $0.2894(0.0002)$ & & \\
\hline 2.7 & 16 & $\mathrm{am}$ & $12.7(0.5)$ & $2.31(0.01)$ & $1.016(0.002)$ & $0.6042(0.0006)$ & $0.4272(0.0003)$ & $0.3412(0.0002)$ & $0.3004(0.0001)$ & $0.2883(0.0001)$ \\
\hline 2.7 & 16 & $\mathrm{fc}_{\mathrm{C}}$ & $12.9(0.6)$ & $2.32(0.01)$ & $1.017(0.002)$ & $0.6044(0.0006)$ & $0.4273(0.0003)$ & $0.3413(0.0002)$ & $0.3004(0.0001)$ & $0.2883(0.0001)$ \\
\hline
\end{tabular}

Table 3: The four-momentum-space ghost propagator $G(k)$ [see eq. (20)] as a function of $N k_{4}$. In all our runs we set $k=\left(0,0,0, k_{4}\right)$. Clearly the same value of $N k_{4}$ for different lattice sizes $N$ does not correspond to the same lattice momentum $p_{4}$ [see eq. (10)]. Since we use periodic boundary conditions, only data for $N k_{4} \leq N / 2$ are reported here. Two different types of statistics are considered: "am" and "fc". (For a definition see the last paragraph in Section 1.) Error bars (in brackets) are one standard deviation. 


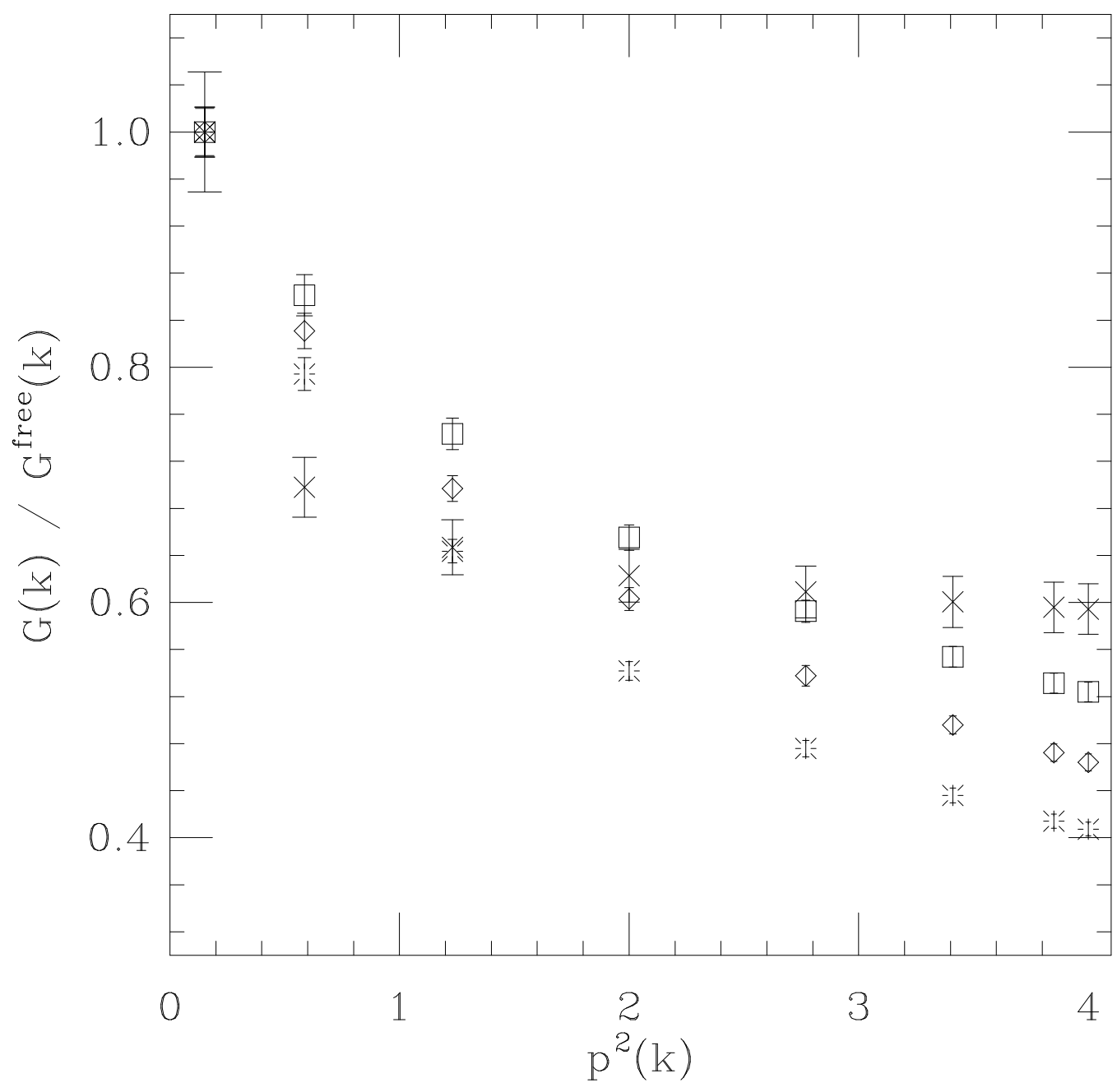

Figure 3: Plot of the four-momentum-space ghost propagator $G(k)$ [see eq. (20)] divided by the corresponding free propagator $G^{\text {free }}(k)$ [see eq. (25)] as a function of $p^{2}(k)$ [see eq. (10)]. In all our runs we set $k=\left(0,0,0, k_{4}\right)$. Since we use periodic boundary conditions, only data for $k_{4} \leq 1 / 2$ are reported here. The data, normalized to one at the smallest nonzero momentum $k_{4}=1$, correspond to $V=16^{4}$ at $\beta=0(\square), \beta=0.8(\diamond), \beta=1.6(*), \beta=2.7(\times)$. Here we consider the "am" statistics (for a definition see the last paragraph in Section 1.) Error bars are one standard deviation. 


\begin{tabular}{||c|c|c||}
\hline \hline$\beta$ & $N$ & $s$ \\
\hline 0.0 & 4 & $1.35(0.03)$ \\
\hline 0.0 & 6 & $1.27(0.01)$ \\
\hline 0.0 & 8 & $1.23(0.01)$ \\
\hline 0.0 & 10 & $1.187(0.009)$ \\
\hline 0.0 & 12 & $1.166(0.009)$ \\
\hline 0.0 & 14 & $1.135(0.001)$ \\
\hline 0.0 & 16 & $1.11(0.02)$ \\
\hline \hline 0.8 & 8 & $1.27(0.01)$ \\
\hline 0.8 & 12 & $1.18(0.01)$ \\
\hline 0.8 & 16 & $1.13(0.02)$ \\
\hline \hline 1.6 & 8 & $1.30(0.01)$ \\
\hline 1.6 & 16 & $1.17(0.02)$ \\
\hline \hline 2.7 & 8 & $1.36(0.03)$ \\
\hline 2.7 & 12 & $1.27(0.03)$ \\
\hline 2.7 & 16 & $1.27(0.03)$ \\
\hline \hline
\end{tabular}

Table 4: Estimate of the pole of the ghost propagator $G(k)$ [see eq. (20)] as the momentum goes to zero. The values of the exponent $s$ [see eq. (27)] are obtained from the data corresponding to the two smallest momenta available for each lattice size (Table 3, average "am"). Errors bars (in brackets) come from propagation of the statistical error on ghost propagator. 